\title{
DOI: https://doi.org/10.46296/rc.v2i3.0006
}

\section{Innovación empresarial y tecnologías de la información y comunicación universitaria}

\section{Business innovation and university information and communication technologies}

\author{
Autor: Chiriboga-Mendoza Fidel Ricardo \\ Universidad Laica Eloy Alfaro de Manabí (ULEAM). Manta, Ecuador. \\ fchiriboga35@gmail.com \\ Autor: Zambrano-Pilay Enrique Cristóbal \\ Universidad Laica Eloy Alfaro de Manabí (ULEAM). Manta, Ecuador. \\ eczp_1997@hotmail.com \\ Autora: Aguaiza-Tenelema Jessenia Maritza \\ Universidad Laica Eloy Alfaro de Manabí (ULEAM). Manta, Ecuador. \\ jesseniaaguaiza19@hotmail.com
}

\begin{abstract}
RESUMEN
La innovación empresarial es un método que desde siempre ha sido forma de atraer a nuevos consumidores, pero en la actualidad el concepto de innovación empresarial se ha visto transformado con la aparición de herramientas como la denominada tecnología de la información y comunicación, a nivel Latinoamericano es cada vez más común ver a empresas que basan su supervivencia en la utilización de herramientas tecnológicas, aunque muchas veces el uso de estas no salga bien.
\end{abstract}

Palabras claves: innovación empresarial, empresa, herramientas tecnológicas.

\begin{abstract}
Business innovation is a method that has always been a way of attracting new consumers, but nowadays the concept of business innovation has been transformed with the appearance of tools such as the so-called information and communication technology, in Latin America it is It is increasingly common to see companies that base their survival on the use of technological tools, although many times their use does not go well.
\end{abstract}

Keywords: business innovation, company, technological tools. 


\section{INTRODUCCIÓN}

La innovación empresarial es un recurso importante para llamar la atención de nuevos consumidores, el concepto de innovación ha ido cambiando con el paso de los años y mucho más en la actualidad con la globalización del internet y es que con la evolución del internet y la evolución de las tecnologías de información y comunicación muchas grandes, medias y pequeñas empresas han visto en estas herramientas una manera de expandir sus alcances, sus productos o sus servicios, a nivel latinoamericano según menciona Pérez Claudia (2018) La innovación empresarial es una tendencia indispensable para la competitividad en el mercado actual que está lleno de crecientes exigencias, ejemplo de esto es Colombia o Nicaragua, siendo el primero un gran expositor del uso adecuado de tanto las herramientas de información y comunicación y el segundo un buen ejemplo del uso de la innovación empresarial.

\section{MATERIALES Y MÉTODOS}

\subsection{La innovación empresarial y su impacto.}

La innovación empresarial ha ido ganando terreno con el surgimiento del internet y cada vez son más las empresas que se alejan de sus estilos de crecimiento antiguos apostando a las nuevas tecnologías, a nivel latinoamericano, esta innovación va directamente relacionadas con pequeñas y medianas empresas que quieren implantar su propia identidad y así entrar a un sistema de mercado que cada vez se vuelve más exigente.

Para Arechavala Ricardo et, al. (2016) La internacionalización de tanto las pequeñas y medianas empresas latinoamericanas es la forma más eficaz de lograr una innovación y competitividad y así generar oportunidades en mercados fuera de su área de alcance, también se indica que para lograr este cometido es necesario acceder a nuevos conocimientos que otorgan a la empresa una capacidad dinámica para sostener un proceso innovador, ante esto discrepan Vélez Olga et, al. (2018) quienes ven una mejor oportunidad de innovación la asociatividad y el liderazgo ambidiestro alegando que los usos de estas herramientas otorgan mejores resultados a las pequeñas y medianas empresas, un caso de innovación y expansión si necesidad de la internacionalización es 
Nicaragua, que ha estudiado como propulsar la economía de las pequeñas y medianas empresas (PYME) sin la necesidad de intentar entrar a competir en mercados internacionales, así lo menciona Ríos Jeferson (2016), uno de los factores que ha logrado impulsar la innovación de empresas en Nicaragua es la asociatividad en el caso de pequeñas y medianas empresas con fines agrícolas que ven en las universidades Nicaragüenses una ayuda significativa ya sea con personal o capacitaciones que han servido para lograr extraer mejores productos y de mejor calidad siendo de gran ayuda al crecimiento del mercado local que a su vez genera empleos para los habitantes de localidades cercanas a estas empresas.

\subsection{Innovación en tecnologías de información y comunicación.}

Al igual que en la innovación empresarial la innovación en tecnologías de información y comunicación son de gran ayuda a las PYMES, brindando un amplio abanico de oportunidades que les puede permitir llegar a más consumidores, para Bernal Mary y Rodríguez Diego (2018) el uso de las tecnologías de información y comunicación es una alternativa para las empresas con fines de optimización y creación de nuevos servicios o productos lo que la convierte en una excelente herramienta que influye de manera positiva a la empresa, ese es el motivo por el cual cada vez más empresas apuestan por un modelo más tecnológico y eficiente al momento de administrar sus empresas, para Ochoa Josefina, Parada Elva y Verdugo María (2017) La innovación en las actuales empresas son de suma importancia para que las mismas sobrevivan en el exigente mercado donde se desenvuelven con el fin de garantizar su supervivencia las empresas hacen uso de las tecnologías de información y comunicación (TIC), aunque cierto es que las empresas que desarrollan innovaciones a base de las TIC se ven optimizadas y competentes no siempre el uso de estas tecnologías no garantizan su éxito, un ejemplo de resultados positivos del uso de las TIC es Colombia que según menciona Botello Héctor (2015) se ha estudiado el impacto delas TIC en alrededor de 17.000 compañías colombianas, que dio como resultado que aquellas empresas que adoptaron TIC poseen un numero de venta por trabajador de un 4,3\% por encima del promedio lo que valida la importancia de herramientas tecnológicas para la evolución de 
una empresa, lo que a simple vista demuestra que apostar por el uso de la tecnología a favor de la evolución de un emprendimiento es una acierto mucho más ahora en los tiempos que transcurren, dándole a Latinoamérica una oportunidad de adentrarse en el mercado y asentar las bases nuevas y futuras empresas.

\subsection{El impacto del uso innovación empresarial y de las TIC en}

\section{Latinoamérica.}

Es acertado mencionar que cada vez es más común la adopción de herramientas poco tradicionales, esto se debe a que se ve en la innovación y en las nuevas tecnologías las empresas ven una oportunidad de generar un impacto más notorio en el consumidor para que los logre solidificar en el mercado, en el contexto latinoamericano se puede mencionar que las posibilidades de una innovación o del uso de las TIC es limitado, sin embargo, se sigue buscando normalizar este tipo de herramientas para potenciar el mercado de Latinoamérica, un ejemplo del buen uso de las herramientas que han sido mencionadas es Argentina exactamente en la localidad de Córdoba que según mencionan Jones Carola, Motta Jorge y Alderete María (2016) Se realizó estudios del cómo afecta la innovación y el uso de las TIC en las Mipymes comerciales determinan que adoptar innovaciones tales como el comercio electrónico afectan positivamente a todas las empresas que emplean estas herramientas, teniendo en cuenta que los factores organizacionales en una empresa afectan que tan bien está siendo llevado a cabo las TIC, se puede concluir que el comercio electrónico en Córdoba, Argentina es un acierto rotundo, al igual que Argentina un país latinoamericano que ha apostado por el uso de las TIC como base de su innovación es Chile así lo mencionan Martínez Carolina y Valenzuela Leslier (2015) quienes a raíz de un estudio acerca del marketing de las medianas y pequeñas empresas recomiendan que se debería desviar los recursos empleados en marketing a mejorar la experiencia de los consumidores que es donde realmente radica la estabilidad de una empresa, la cultura organizacional de la empresa debería trabajar en conjunto con las TIC para desarrollar capacidades tecnológicas que ayuden a la empresa al manejo de la tecnología para a su vez centrar la atención a mejorar la experiencia de los consumidores, cabe mencionar que en el caso de Chile cada vez más se apuesta 
por el marketing en línea aunque según se menciona la experiencia del consumir sigue invariable.

Por el contrario, como se mencionó con anterioridad el uso de las TIC no asegura la efectividad de una empresa, esto se debe a las limitaciones como el dinero puede tener la misma empresa o el poco desarrollo que tienen herramientas como las TIC o la innovación en la localidad de la empresa, un ejemplo del fracaso en el uso de las TIC e innovación empresarial es Ecuador, debido a que en la ciudad de Ambato tal y como lo menciona Quispe Ana et, al. (2017) se desarrolló un estudio con la finalidad de estudiar el impacto de las TIC e innovaciones tomando una muestra de 87 PYMES, en las cuales el uso de las TIC fue ineficiente debido a que su limitación tecnológica y carencia de recursos como software contable fueron un perjuicio para las actividades de las empresas estudiadas también mencionando que las carencias de leyes y acciones del gobierno parar que impulsar el uso eficiente de las TIC es una limitación para que las empresas evolucionen, privándolas del uso de estas tecnologías por temor a fracasar, dejando en claro que las limitaciones presentes en la empresa también son delimitantes en el buen uso de las TIC y que no siempre las mismas deben funcionar.

\section{CONCLUSIONES}

Para concluir, La manera en la que las empresas llegan a sus clientes varia constantemente cada vez las empresas se van alejando de los modos de marketing convencionales dejándoles vía abierta al uso de nuevos modos, y es que con el cambio que las empresas realizan para generar más clientelas también sufren cambios en las herramientas que están a la disposición de las propias empresas para poder llegar a los consumidores, el uso de la tecnología se ha convertido para muchas empresas en la piedra angular de su innovación ya no únicamente en países super desarrollados es común ver cómo usan la tecnología al antojo para generar más clientes, en el caso de Latinoamérica es un acierto remarcar que cada vez es más común ver a empresas hacer uso de las TIC como base de su innovación que con las mismas buscan generar una buena impresión a su consumidores, sin embargo, hay un largo trecho que se 
debe recorrer para generar una base fuerte que permita competir a Latinoamérica con el mercado internacional, a pesar de esto, hay exponentes que se arriesgan al uso de estas herramientas como lo son Colombia, Chile o Argentina que ya están apostando por el mercado en línea y herramientas de tecnología para lograr llegar a más personas siendo muestras que el cambio es posible y que la innovación sigue siendo el motor que podría impulsar a las medianas y grandes empresas, por otra parte hay pruebas de lo que significa arriesgarse al cambio se puede hacer mención a Ecuador que debido a sus limitaciones monetarias el uso de las TIC demostrando que el uso de estas herramientas no necesariamente significa que traerán cambios positivos.

\section{REFERENCIAS}

Bernal, O. I. V., Ríos, J. A. B., Giraldo, J. A. L., \& Vargas, F. J. A. (2018). Asociatividad empresarial y liderazgo ambidiestro como generadores de innovación. Revista de ciencias sociales, 25(2), 51-72.

Jiménez, M. C. B., \& Ibarra, D. L. R. (2018). Las tecnologías de la información y comunicación como factor de innovación y competitividad empresarial. Scientia et technica, 24(1), 85-95.

Jones, C., Motta, J., \& Alderete, M. V. (2016). Gestión estratégica de tecnologías de información y comunicación y adopción del comercio electrónico en Mipymes de Córdoba, Argentina. Estudios gerenciales, 32(138), 4-13.

Peñaloza, H. A. B., Avella, A. C. P., \& Pacheco, O. E. C. (2015). Análisis empresarial de la influencia de las TIC en el desempeño de las empresas de servicios en Colombia. REVISTA VIRTUAL universidad Católica del Norte, 2(45), 3-15.

Pérez, C. M. (2018). Innovación empresarial al servicio de la micro y pequeña empresa nortesantandereana: por la competitividad regional. ECONÓMICAS CUC, 40(1).

Quispe-Otacoma, A. L., Padilla-Martínez, M. P., Telot-González, J. A., \& Nogueira-Rivera, D. (2017). Tecnologías de información y comunicación en la gestión empresarial de pymes comerciales. Ingeniería Industrial, 38(1), 81-92.

Ríos Quiñonez, J. (2016). La innovación y su impacto económico en las empresas de Nicaragua. 
Ruiz, J. O., Ruiz, E. L. P., \& Tapia, M. L. V. (2017). El uso estratégico de las tecnologías de información y comunicación para incrementar la innovación en las empresas. Red Internacional de Investigadores en Competitividad, 2(1).

Valenzuela Fernández, L., \& Martínez Troncoso, C. (2015). Orientación al Cliente, Tecnologías de Información y Desempeño Organizacional: Caso empresa de consumo masivo en Chile.

Vargas, R. A., Andrés, M. F., Agramunt, L. F., \& Pineda, J. M. B. (2016). Gestión del conocimiento en los procesos de internacionalización de empresas Latinoamericanas de base tecnológica. Universitas: Gestão e TI, 6(1). 